\title{
L'EXHIBITION ET LA NÉGOCIATION DU SAVOIR DANS LES PRATIQUES DÉFINITOIRES : L'INTERACTION AUTOUR DU SYNDROME DE FATIGUE CHRONIQUE DANS UN GROUPE D'ENTRAIDE
}

Elwys De Stefani, Paul Sambre

Armand Colin | « Langages »

2016/4 N²04 | pages 27 à 42

ISSN 0458-726X

ISBN 9782200930424

Article disponible en ligne à l'adresse :

http://www.cairn.info/revue-langages-2016-4-page-27.htm

\section{Pour citer cet article :}

Elwys De Stefani, Paul Sambre, «L'exhibition et la négociation du savoir dans les pratiques définitoires : l'interaction autour du syndrome de fatigue chronique dans un groupe d'entraide », Langages 2016/4 (N²04), p. 27-42.

DOI 10.3917/lang.204.0027

Distribution électronique Cairn.info pour Armand Colin.

(C) Armand Colin. Tous droits réservés pour tous pays.

La reproduction ou représentation de cet article, notamment par photocopie, n'est autorisée que dans les limites des conditions générales d'utilisation du site ou, le cas échéant, des conditions générales de la licence souscrite par votre établissement. Toute autre reproduction ou représentation, en tout ou partie, sous quelque forme et de quelque manière que ce soit, est interdite sauf accord préalable et écrit de l'éditeur, en dehors des cas prévus par la législation en vigueur en France. Il est précisé que son stockage dans une base de données est également interdit. 


\section{L'exhibition et la négociation du savoir dans les pratiques définitoires : l'interaction autour du syndrome de fatigue chronique dans un groupe d'entraide}

\section{INTRODUCTION}

Cet article se penche sur des pratiques définitoires observables dans un contexte spécifique : des groupes d'entraide animés par des personnes souffrant du syndrome de fatigue chronique (SFC), également connu sous le nom d'encéphalomyélite myalgique $(E M)^{1}$. Sur le plan clinique, cette pathologie se caractérise par un état d'épuisement intense et persistant qui n'est pas atténué par le sommeil, et qui est souvent accompagné d'autres plaintes - douleurs musculaires, troubles digestifs, difficultés cognitives, etc. - entraînant une réduction importante de toute activité et pouvant aller jusqu'à l'incapacité de travail permanente (Fukuda et al. 1994). Il n'existe à ce jour aucun examen médical permettant aux médecins de poser un diagnostic positif. Le SFC est dès lors diagnostiqué en excluant une série d'autres pathologies qui présentent une fatigue excessive comme symptôme cardinal (p. ex. tumeurs, burnout, dépression). L'absence de biomarqueurs permettant de dépister le SFC engendre de vifs débats au sein même du corps médical, mais aussi parmi les malades, concernant la définition de cette pathologie. Alors que

\footnotetext{
1. Les difficultés de conceptualisation de cette maladie se manifestent déjà dans la grande variété de noms qui sont utilisés pour décrire des problèmes de santé se manifestant principalement par une fatigue chronique et handicapante (voir Sharpe et al. 1991). Récemment, l'Institute of Medicine des États-Unis a publié un rapport (IOM 2015) proposant un changement de nom pour le syndrome de fatigue chronique, désormais nommé Systemic exertion intolerance disease ('maladie systémique d'intolérance à l'effort').
} 
certains médecins, en accord avec l'International Classification of Diseases (ICD10), y reconnaissent une pathologie d'origine somatique (neurologique), pour d'autres, il s'agirait d'une maladie d'ordre psychique. Par conséquent, le SFC est souvent décrit comme une maladie « contestable " (Bülow 2004) ou « discutable ( Tucker 2004), présentant un « dilemme diagnostique» (Sachs 2001). Cette inconsistance définitoire se révèle extrêmement problématique pour les personnes recevant un tel diagnostic : le fait de souffrir de symptômes invalidants sans en connaître l'étiologie produit des remises en question, voire des contestations du diagnostic. C'est dans les groupes d'entraide que les souffrants croisent leurs points de vue sur différents aspects de la maladie. Nos analyses se basent sur un corpus de sept rencontres d'un groupe d'entraide flamand (environ $3 \mathrm{~h}$ chacune). Pendant ces rencontres, réunissant une trentaine de personnes, différents formats de participation sont observables, allant de présentations données par des conférenciers invités à des discussions en petits groupes. Nous appliquons à ces données l'instrumentaire analytique qu'offre l'analyse conversationnelle, qui vise à décrire les pratiques et les méthodes que les participants mettent en œuvre dans leurs interactions sociales. Cela nous permet d'appréhender la définition non pas comme une opération cognitive aspirant à établir un équivalent informationnel d'un terme donné mais comme un ensemble de pratiques interactionnelles que les participants déploient au cours de leurs interactions et qu'ils peuvent mobiliser avec différentes finalités.

Se fondant sur des données empiriques, non sollicitées, enregistrées dans l'habitat naturel de leur occurrence, notre recherche propose une approche nouvelle et originale du SFC qui, jusqu'ici, a été abordé au travers de questionnaires, d'interviews ou de groupes de parole tant dans les études psychomédicales (Clements et al. 1997) qu'en sciences humaines et sociales, notamment en analyse du discours (Grue 2014a, 2014b) et en psychologie discursive (HortonSalway 2001, 2002). Avant de nous pencher sur les données, nous fournissons au paragraphe suivant les repères épistémologiques nécessaires qui permettront de mieux situer notre recherche (voir aussi De Stefani, Sambre \& Van De Mieroop 2016).

\section{LA DÉFINITION ENTRE PHILOSOPHIE ET LINGUISTIQUE}

Le concept de définition est au cœur des préoccupations des philosophes (du langage) et des linguistes et s'inscrit dans une ligne de pensée millénaire. $\mathrm{Si}$ pour Platon, la définition est un « instrument de l'action dialectique » (Sambre, 2005 : 20), chez Aristote, elle devient « une quasi-démonstration de l'essence » d'un objet (op. cit. : 31), comme le rappelle le raisonnement axiomatique ou syllogistique destiné à influencer toute la pensée médiévale jusqu'au renouveau opéré par Leibniz. Pour Aristote, une définition (ỏpos 'hóros') est un raisonne-

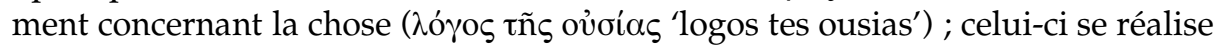
dans une parole qui met en évidence la désignation (oquaivov 'sémainon') de 
son essence ( $\tau$ ò $\tau$ í $\tilde{\eta} v$ عĩvol 'to ti en einai') ${ }^{2}$. À partir du XIX ${ }^{\mathrm{e}}$ siècle, les philosophes logiciens se sont davantage intéressés au lien qui existerait entre un nom et l'objet qu'il désigne (notamment Mill 1843). Cela a mené, d'une part, à une conception mathématique de la définition, p. ex. lorsque A. Whitehead et B. Russell (1910-1913) appréhendent celle-ci comme un rapport d'équivalence entre un definiens et son definiendum ; d'autre part, à la distinction conceptuelle que G. Frege (1892) opère entre Sinn ('sens') et Bedeutung ('dénotation').

En linguistique, la définition est traditionnellement traitée comme un phénomène $\mathrm{d}^{\prime}$ ordre sémantique : un mot peut être défini de manière intensionnelle par une analyse en sèmes (Pottier 1963), de manière extensionnelle en mobilisant des critères de prototypicité (Kleiber 1990), etc. La définition constitue en outre un problème pratique des lexicographes et des terminographes, qui collectent et traitent des termes utilisés dans des domaines de spécialisation (p. ex. en médecine). Les pratiques des lexico- et terminographes réifient une vision intensionnelle, logique et universelle de la définition, intrinsèquement liée à l'objectif (ontologique) de la lexicographie (Robinson 1962). La définition linguistique est souvent perçue comme une explication per genus proximum et differentia specifica ${ }^{3}$. Détachée de tout contexte d'élocution, elle a pour finalité une classification taxonomique des termes basée sur un principe de différentiation et d'abstraction. R. Martin (1990 : 86-87) oppose cette « définition conventionnelle», stipulatoire, qui appartiendrait à un locuteur idéal, à une « définition naturelle ", située et contextualisée, qui émanerait des locuteurs. Ce que R. Martin appelle une « définition naturelle » relève pour d'autres auteurs du domaine de la description, qui rassemblerait les traits contextuels (et non pas universels) de la chose nommée (Rey-Debove, 1971 : 183). La distinction introduite par R. Martin (1990) effleure la dimension située et praxéologique de la définition qui a longtemps été négligée par la linguistique. Pourtant, la possibilité d'appréhender la définition comme une pratique plutôt que comme une équation sémantique est bien présente dans les auteurs antérieurs. Ainsi, si Y. Bar-Hillel (1954) décrit la dimension indexicale des définitions, c'est surtout le deuxième L. Wittgenstein qui avance tout un éventail de réflexions qui remettent en question la conceptualisation de la définition comme un ensemble de traits nécessaires et suffisants. La définition se manifeste, au contraire, dans une pratique sociale (Lebensform 'forme de vie'), plus précisément dans un jeu de langage ou Sprachspiel (Wittgenstein, 1953 : § 23 ; voir aussi § 68). Le fondateur de l'ethnométhodologie, H. Garfinkel, emprunte précisément à Y. Bar-Hillel la notion d'indexicalité (Garfinkel, $1967: 4$ ) et l'on connaît l'influence que le Wittgenstein des Philosophische Untersuchungen a exercé sur les ethnométhodologues et les analystes de la conversation. Il est d'autant plus surprenant que le domaine de la sémantique - dans lequel la définition s'inscrit traditionnellement en linguistique - ne soit traité que tardivement par des linguistes d'orientation interactionnelle (voir Deppermann 2011a, 2011b).

2. Voir p. ex. Aristote, Topiques, I, 5, $101 \mathrm{~b} 38$.

3. Voir p. ex. Aristote, Seconds analytiques, II, 13, 96b25-97b15. 
Si, en suivant L. Wittgenstein, nous considérons la définition comme une pratique ordinaire, celle-ci devient descriptible comme un accomplissement réalisé de différentes manières, p. ex. par monstration, comparaison, paradigmatisation, catégorisation, etc. (voir les articles de Deppermann ; Greco ; Traverso et Ravazzolo dans ce numéro).

\subsection{Pratique définitoire, catégorisation et exhibition du savoir}

Dans les groupes d'entraide, ainsi que dans le domaine médical en général, les pratiques définitoires ne constituent jamais une fin en soi. Au contraire, elles répondent à des exigences pratiques des participants. Ainsi, le fait de définir le SFC comme une maladie neurologique vs psychique entraîne des catégorisations diverses du souffrant (comme « malade somatique » ou « malade psychique ») associées à des différences en termes de category-bound activities ('activités liées à la catégorie' ; Sacks 1972) et de category-bound predicates ('caractéristiques liées à la catégorie" ; Watson 1978). Par exemple, un souffrant perçu comme un « malade psychique » peut être vu comme quelqu'un qui suit un traitement psychiatrique (activité), qui est trop préoccupé par sa santé (caractéristique), etc. La définition de la maladie oriente, par conséquent, les examens et traitements subséquents au diagnostic. Ainsi, dans le cas du SFC, la définition de la maladie se confond avec la description de son étiologie et équivaut à un diagnostic. C'est notamment son acception psychique que les participants des groupes d'entraide remettent en question. Dans l'extrait suivant, Bert formule cette catégorisation, que beaucoup de membres du groupe d'entraide critiquent :

Extrait 1. CFS 3165_2, 03:02-03:10 ${ }^{4}$

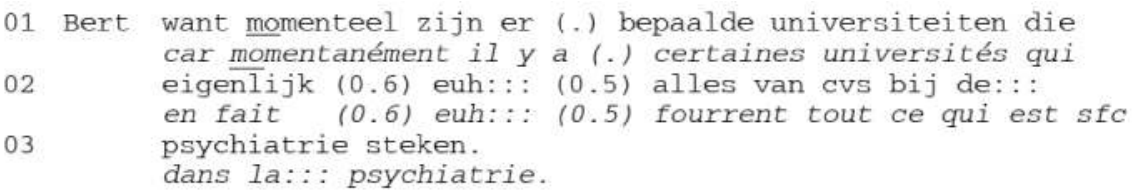

Cet extrait nous permet de souligner une dimension fondamentale des pratiques définitoires, à savoir leur ancrage épistémique : en effet, les participants peuvent assumer la responsabilité de leurs définitions ou attribuer cette responsabilité à d'autres acteurs (absents), comme « certaines universités » (1. 1). Ainsi, en s'engageant dans une pratique définitoire les participants déploient un savoir ou, au contraire, manifestent l'absence d'un savoir, voire un accès à un « autre » savoir. L'exhibition et la négociation du savoir ont été décrites comme une dimension fondamentale de la rencontre sociale : selon J. Heritage et G. Raymond (2005) et J. Heritage (2012), les interactants seraient constamment en train de déployer

\footnotetext{
4. Les données ont été transcrites en suivant les conventions de transcription jeffersoniennes (Jefferson 2004). Une version abrégée de ces conventions se trouve en annexe. Les traductions en français sont le plus proche possible du néerlandais parlé : cette fidélité à l'original peut parfois se refléter dans l'utilisation de tournures peu usuelles en français.
} 
leur savoir. Ils le font en s'appuyant sur ce que les auteurs appellent «statut épistémique » (epistemic status) et en endossant un certain " positionnement épistémique » (epistemic stance). Le statut épistémique est associé de façon normative à des identités sociales et rendu pertinent par les interactants, qui s'orientent manifestement vers un déséquilibre du savoir (Deppermann 2015). Ainsi, en matière de maladies, un médecin posséderait un statut épistémique supérieur à celui d'un patient. En d'autres termes, il « en sait plus » qu'un patient. En revanche, lors d'une interaction, un patient, un médecin, ou tout autre locuteur, peut exhiber du savoir (knowledge), endossant ainsi un positionnement épistémique du type $k+$ (knowledge+), ou alors rendre reconnaissable une absence de savoir $(k-)$, typiquement par la formulation d'une question. On sait que, dans les interactions médecin/patient, les participants déploient des orientations asymétriques vers différentes formes de savoir (Ariss 2009, Lehtinen 2013, De Fornel \& Verdier 2014, Deppermann 2015). De façon similaire, dans les rencontres analysées dans cet article, les interactants s'orientent massivement vers différents domaines de savoir, liés à l'expérience de leur propre corps, à des connaissances médicales conventionnelles et non conventionnelles, à des compétences en matière de législation sur les maladies chroniques, d'assurances, etc. Dans les analyses qui suivront, nous observerons comment une asymétrie du savoir est appréhendée par les membres du groupe d'entraide observé.

\subsection{Le diagnostic comme pratique définitoire}

L'interaction médecin/patient a fait l'objet de bon nombre d'études en analyse conversationnelle à partir des années $80^{5}$. Depuis ces travaux pionniers, la recherche s'est passablement différenciée : V. Gill et F. Roberts (2013 : 578-579) identifient trois domaines d'étude qui seraient privilégiés à l'heure actuelle, à savoir :

- les interactions médecin/patient en médecine générale (cabinets), mais aussi en médecine spécialisée (hôpitaux et cliniques) ;

- les interactions patient/patient et celles entre patients et praticiens en médecine non conventionnelle ;

- les interactions entre médecins, professionnels du domaine médical, etc.

Le terrain que nous étudions dans cet article peut être inséré dans la deuxième ligne de recherche. Toutefois, il n'existe à ce jour que peu d'études analysant les interactions dans des groupes de parole consacrées à des thématiques médicales. On ne peut citer dans ce domaine que les études sur des thérapies psychologiques de groupe (MacMartin \& LeBaron 2006, Halonen 2008) et les recherches approfondies de I. Arminen (1998) sur l'organisation de l'interaction dans un groupe d'entraide spécifique, les alcooliques anonymes. Dans ces groupes, la narration de sa propre expérience avec la maladie constitue un travail thérapeutique

5. Pour une présentation des travaux portant sur l'interaction médecin/patient dans une perspective conversationnelle, voir Heritage \& Maynard (2006) et Gill \& Roberts (2013). 
qui vise et souvent atteint la guérison ; le partage des expériences permet aux participants, entre autres, de constituer une identité collective $\mathrm{d}^{\prime}$ " alcooliques en voie de guérison » et de construire une moralité de solidarité et de soutien réciproque (Arminen 1998). Les alcooliques anonymes s'orientent vers un format de participation qui favorise la narration et qui inhibe la contestation de la part des autres participants (op. cit. : 49-79). Les données sur lesquelles nous basons notre article se différencient des interactions étudiées par I. Arminen à deux égards. D'une part, les groupes d'entraide SFC ne poursuivent pas d'objectif thérapeutique : en d'autres termes, la discussion et la narration d'expériences personnelles n'ont pas pour objectif de guérir la maladie, mais de discuter des problèmes pratiques que la maladie entraîne dans le vécu quotidien. D'autre part, nous observons une plus grande diversité de formats de participation (voir $\S 1$ ). Les groupes d'entraide que nous observons offrent aux participants la possibilité de déployer leurs expériences et leurs différentes expertises autour du SFC. $C^{\prime}$ est le lieu où les diverses définitions, parfois discordantes, émergent au cours de l'interaction. C'est aussi le lieu où les souffrants formulent leur « diagnostic profane » (lay diagnosis, Beach 2001) au travers duquel les participants rendent leur maladie descriptible (accountable) et remédient à l'absence d'un diagnostic professionnel, qui serait basé sur des résultats biomédicaux positifs.

\section{LES DIMENSIONS PRAXÉOLOGIQUES ET ÉPISTÉMIQUES DES PRATIQUES DÉFINITOIRES}

Nous proposons ici une analyse contextualisée et située des pratiques définitoires, en analysant notamment les cas où une pratique définitoire émerge de façon non responsive, i.e. sans être projetée par une question ou par une autre action préalable. Nous examinons donc les cas où un locuteur accomplit une pratique définitoire en première position séquentielle, revendiquant par là un savoir spécifique. Nous décrivons deux manières différentes de réceptionner une telle revendication, à savoir en exprimant un accord (§ 3.1) ou alors en la contestant par un désaccord $(\S 3.2)$.

\subsection{Manifestations d'accord avec le savoir exhibé}

Le premier extrait de cette section a été récolté lors d'une discussion de cinq personnes sur les coûts engendrés par la maladie. Les participants viennent de parler du problème de la reconnaissance du SFC en tant que maladie. Après une pause (1. 1), Marie s'auto-sélectionne : 
Extrait 2. CFS 111029_3 (19:59-20:15)

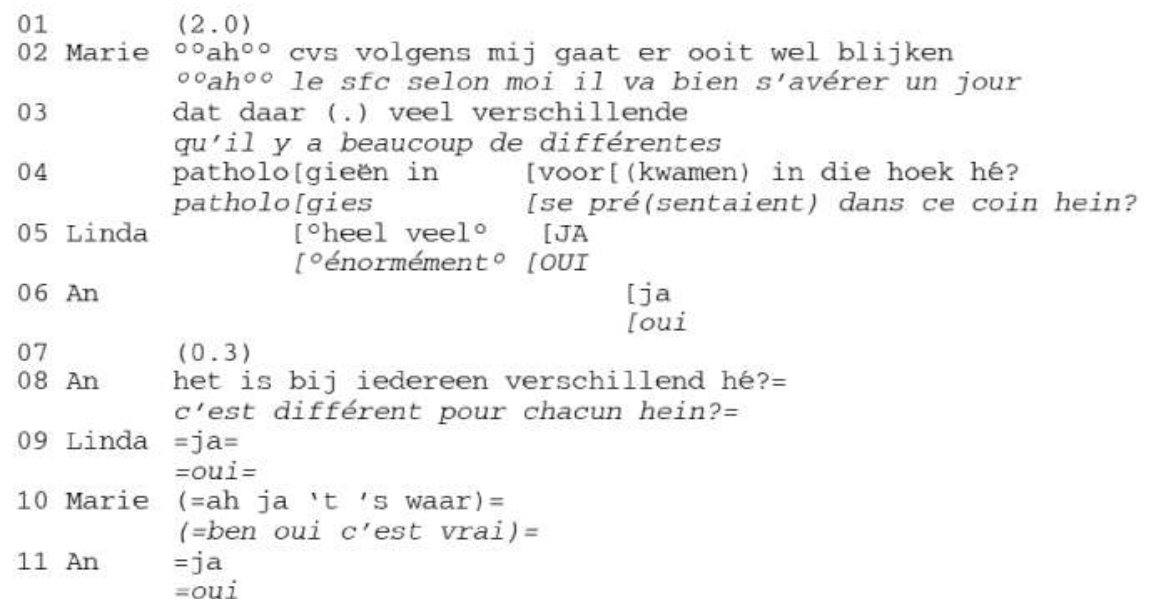

Marie prend la parole à un moment où le topic précédent sur la reconnaissance du SFC est possiblement clôturé. Elle initie son tour de parole par l'élément référentiel («sfc ») projetant ainsi dès le départ qu'elle va dire quelque chose à propos de ce référent. Ce qui suit pourrait être décrit comme une définition du SFC que Marie décrit comme un ensemble de «pathologies différentes » (1. 3-4). En fait, de cette manière, Marie remet en question l'existence même d'un référent unique et homogène auquel s'appliquerait le terme de SFC. Les co-participantes s'orientent très tôt vers l'émergence de cette définition. Alors que l'action de Marie devient progressivement interprétable comme une définition, Linda manifeste de façon de plus en plus incarnée son accord, par des hochements de tête (non reproduits) et aussi par la parole, lorsqu'elle intervient en chevauchement, tout en recyclant l'adverbe " veel » ('beaucoup'), que Marie vient d'utiliser (1. 3), et en formulant un « OUI » (1. 5). Les autres participants (à l'exception de Steven qui s'est momentanément désengagé de la conversation) manifestent également leur accord avec la position de Marie : An produit un « oui » (1. 6) alors que Lieve hoche la tête (non reproduit dans la transcription).

Alors qu'à la ligne 7 le topic risque de s'estomper, An affiche de nouveau son accord avec la définition avancée, cette fois-ci en reformulant la position de Marie : « c'est différent pour chacun hein? » (1. 8). Dans cette reformulation, An reprend la notion de différence présente dans la définition de Marie (1.3) et en fait un aspect qui serait observable dans les expériences des souffrants ( " chacun ») - alors que Marie présentait la « différence » comme un problème de catégorisation des pathologies recevant l'étiquette de SFC. Cette reformulation est à son tour suivie de manifestations d'accord de la part de Linda (1. 9) et de Marie (1. 10).

Cet extrait illustre un cas où les participants sont fortement orientés vers l'exhibition d'un accord avec une pratique définitoire accomplie en première 
position. Cela vaut non seulement pour les récipiendaires mais aussi pour celles qui initient et/ou reformulent la définition : en effet, tant Marie (1. 4) qu'An (1. 8) terminent leurs tours de parole par la tag question « hé? » ('hein?'), qui projette un accord comme réponse préférentielle.

\subsection{Les définitions contestées}

Contrastons l'extrait présenté au paragraphe précédent avec des cas où une définition initiale est contestée. Nous allons nous focaliser dans cette section sur deux manières d'exprimer un désaccord : dans le premier cas de figure, le désaccord met en évidence la dimension «subjective » de la définition avancée (§ 3.2.1) ; le désaccord peut aussi être réalisé par une remise en question de l'autorité épistémique du locuteur s'étant engagé dans une pratique définitoire (§ 3.2.2).

\subsubsection{L'accountability individuelle des pratiques de définition}

Lors d'un travail en groupes restreints autour d'une table, les participants évoquent un livre dans lequel une femme raconte comment elle a pu vaincre le SFC. Les participants ont exprimé des avis partagés sur ce livre : certains l'apprécient, d'autres estiment que ce que l'auteur décrit n'a rien à voir avec le SFC ; ils y reconnaissent plutôt la conséquence d'une vie frénétique que l'auteur dit avoir menée avant les début de ses plaintes. Nous rejoignons l'interaction au moment où Greet avance une description du SFC :

\section{Extrait 3. CFS 4A1115_1 (08:58-10:17)}

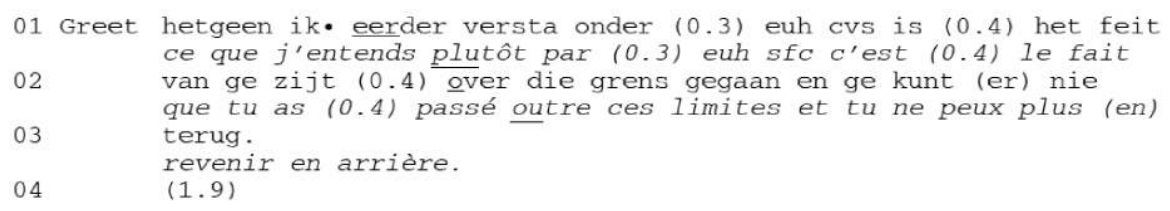

On notera que Greet présente dès le départ ce qu'elle va dire comme reflétant un point de vue personnel (« ce que j'entends plutôt »,1. 1). Elle formule ensuite une description du SFC, qu'elle conçoit comme étant la conséquence d'avoir " passé outre ces limites » sans avoir la possibilité de " revenir en arrière » (1. 2-3). Cette description est suivie, après une pause relativement longue, de différentes prises de parole : Aline se réfère à l'auteur du livre dont il a été question précédemment, en disant qu'elle a dépassé les limites (1. 5). Jeroen s'auto-sélectionne au même moment et amorce un tour qui manifeste un désaccord avec la définition que Greet vient de formuler (1. 6): 


\section{Extrait 4. CFS 4A1115_1 (08:58-10:17)}

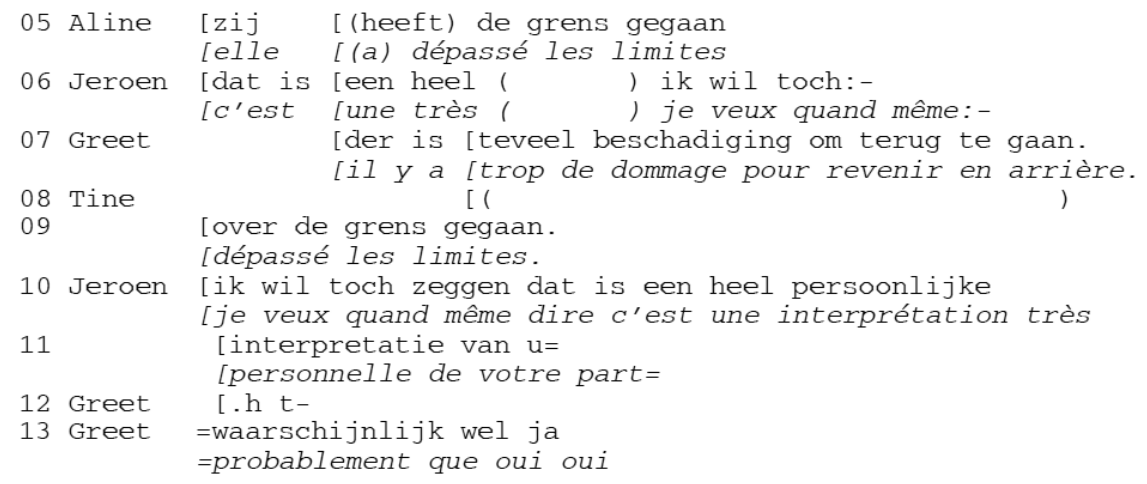

En chevauchement avec Jeroen, Greet ajoute un élément explicatif en disant qu'il y a « trop de dommage » (1. 7) pour « revenir en arrière ». C'est à la fin de ce tour que Jeroen reformule (en chevauchement avec Tine, 1.9) son désaccord interrompu précédemment. Il structure son tour de manière à mettre en évidence l'argument de son désaccord : en effet, le début de son tour («je veux quand même dire »,1.10) projette au moins deux continuations possibles, à savoir une subordonnée complétive en que ou alors une proposition autonome, i.e. non subordonnée. C'est cette dernière possibilité que Jeroen réalise en disant « c'est une interprétation très personnelle de votre part » (1. 10-11). Le désaccord de Jeroen opère au niveau épistémique, non pas en remettant en question l'autorité épistémique que Greet possède par rapport à l'expérience de son propre corps, mais en exhibant le caractère personnel, individuel et non généralisable de cette expérience. Greet accepte cette objection (1.13), en assumant la responsabilité (accountability) personnelle de ce qu'elle vient de dire. Dans la suite de la conversation, Jeroen et Greet consolident leurs opinions respectives, tout en manifestant $q^{\prime}$ 'ils acceptent le point de vue divergent de l'autre. Cette divergence d'opinions porte sur les causes qui peuvent déclencher le SFC et donc sur son étiologie qui comme nous l'avons expliqué au § 2.1 - est une dimension fondamentale de la définition de cette maladie.

Dans la suite de l'interaction, les participants comparent les problèmes étiologiques du SFC aux difficultés diagnostiques de la douleur chronique. Nous rejoignons la discussion environ 40 secondes plus tard : 
Extrait 5. CFS 4A1115_1 (08:58-10:17)

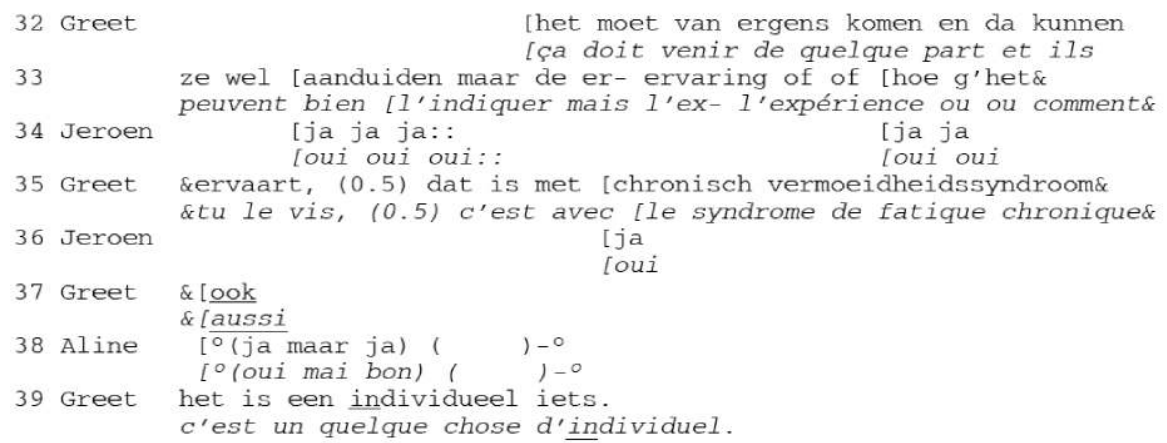

Si la douleur doit bien "venir de quelque part» (1. 32) et que l'origine de la douleur peut être « indiquée » par les médecins (« ils », 1. 33), cela ne dit rien sur la manière dont les personnes la ressentent. C'est en cela que l'expérience de la douleur ressemble à l'expérience de la fatigue : " c'est avec le syndrome de fatigue chronique aussi » (1. 35-37). Cette comparaison de Greet trouve l'accord de Jeroen $(1.34,36)$ et culmine dans une définition du SFC, formulée comme «c'est un quelque chose d'individuel " (1. 39). Alors que, d'un point de vue sémantique, cette définition est très vague, $\mathrm{d}^{\prime}$ un point de vue praxéologique elle est parfaitement adaptée à la position séquentielle qu'elle occupe. En effet, le désaccord initial entre Jeroen et Greet a été transformé peu à peu en un accord : la formulation que Greet produit à la ligne 39 est, grâce à son caractère général, une ressource qui permet de réaffirmer une position désormais partagée par les deux interlocuteurs et de clôturer la séquence de désaccord et le topic en question en formulant une sorte de « vérité générale " (voir Drew \& Holt 1998). Notons enfin que cette définition opère aussi au niveau catégoriel : le SFC n'est pas décrit comme une "maladie », un « syndrome », un « handicap », etc., mais, par le biais d'une substantivation d'un pronom indéfini, comme un « quelque chose » (1. 39).

L'extrait analysé dans cette section a permis d'observer l'émergence et la gestion d'un désaccord relatif à une description ou définition du SFC. Un désaccord donne lieu à une argumentation et à une définition successive qui - du point de vue du contenu - pourrait paraître banale, mais qui, d'un point de vue praxéologique, répond aux besoins interactionnels des participants. Ainsi, la définition " het is een individueel iets " 'c' est un quelque chose d'individuel' (1. 39) permet d'exhiber l'accord retrouvé par deux participants qui affichaient un désaccord initial. Dans cet extrait, la gestion du désaccord se fait sans remettre en cause le statut épistémique des locuteurs.

\subsubsection{La remise en cause de l'autorité épistémique}

Dans la dernière section analytique de cet article, nous illustrons brièvement un cas dans lequel une définition en première position n'est pas suivie d'un 
désaccord exhibé, mais d'une remise en question de l'autorité épistémique du locuteur ayant formulé la définition. Karel parle depuis un moment de son parcours en tant que malade du SFC. Sa longue intervention (non reproduite) culmine dans une pratique définitoire (1. 3-4) :

\section{Extrait 6. CFS 3165_2 (08:45-09:24)}

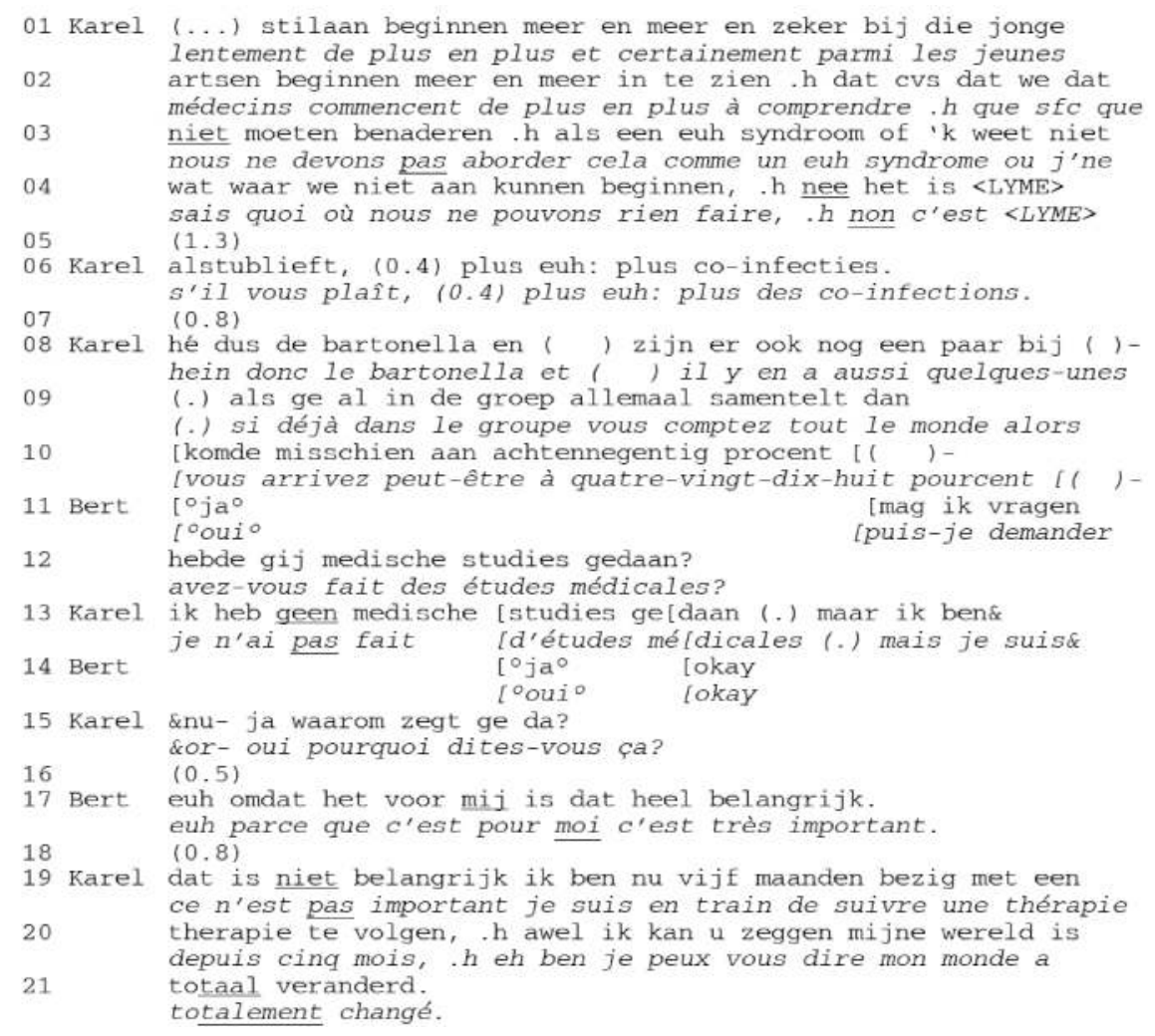

Karel s'oriente vers une pratique définitoire à partir de la ligne 1, lorsqu'il se réfère à une catégorie de personnes ("les jeunes médecins ») qui n'aborderaient désormais plus le SFC comme "syndrome » (1. 3). Alors que, jusqu'ici, Karel semble attribuer cette pratique à une catégorie de personnes qui possèdent une certaine autorité épistémique en la matière, à la fin de la ligne 4, il formule une définition " absolue »: " non c'est $\langle\mathrm{LYME}\rangle$ ». Karel emphatise cette définition, d'une part, par la négation initiale, d'autre part, par une prononciation ralentie du nom de la maladie, ce qui est énoncé également à un volume élevé. Contrairement à ce que nous avons vu dans les extraits précédents, Karel n'obtient aucune manifestation d'accord ou de désaccord (voir la pause à la 1. 5). Face à cette absence de réponse, il étend son tour de parole, modifiant ainsi la définition même : le SFC serait en réalité la maladie de Lyme "plus des coinfections » (1. 6). De nouveau, personne ne s'auto-sélectionne, ce qui donne 
lieu à une nouvelle pause (1. 7). Karel passe ensuite à l'énumération des infections (1. 8) et poursuit en affirmant que " peut-être [...] quatre-vingt-dix-huit pourcent » des membres du groupe souffriraient de la maladie de Lyme (1. 10). $\mathrm{C}^{\prime}$ est à ce moment-là que Bert l'interrompt, en chevauchement : " puis-je demander avez-vous fait des études médicales? » (1. 11-12). En posant cette question, Bert remet en question l'autorité épistémique de Karel : en d'autres termes, il le traite comme quelqu'un qui ne possède pas les connaissances nécessaires et qui, par conséquent, n'a pas le droit d'avancer ce genre de définitions. Karel amorce une réponse (l. 13), mais abandonne celle-ci avant d'adresser lui-même une question à Bert : «pourquoi dites-vous ça? » (1. 15). Avec cette question, Karel exhibe l'analyse en temps réel qu'il est en train de faire de l'interaction dans laquelle il est engagé. Cette action de Karel montre, d'une part, que la question « why that now ? " (Schegloff \& Sacks, 1973 : 299), fréquemment citée dans les travaux conversationnalistes, est une préoccupation constante des interactants. D'autre part, Karel, à son tour, conteste l'action précédente de Bert en traitant sa question (1. 11-12) comme une action qui n'est pas interprétable de façon contingente mais qui nécessite une justification pour son occurrence à ce moment précis de l'interaction. C'est précisément la manière dont Bert répond à la ligne 17, où il précise qu'il s'agit d'une question « importante » pour lui. Aux lignes 19-20, Karel exhibe d'abord son désaccord avec ce que Bert vient de dire («ce n'est pas important»). Cela lui permet, entre autres, de refuser le lien que Bert a établi entre l'appartenance à une catégorie sociale (ceux qui ont fait des « études médicales ») et le droit de s'engager dans des pratiques définitoires. Ensuite, il fournit une preuve empirique, basée sur son expérience personnelle, de la vérité de ses propos : cela fait cinq mois qu'il suit un traitement (que l'on suppose être effectif contre la maladie de Lyme) qui a " totalement changé » son monde (1. 19-21).

\section{CONCLUSIONS}

Les pratiques définitoires se révèlent particulièrement nombreuses dans les entretiens du groupe d'entraide que nous avons observés. Nous avons considéré comme pratique définitoire toute action des participants visant à décrire, caractériser, catégoriser, etc. une maladie dans ses multiples facettes (symptômes, modes de transmission, traitements, etc.) et revendiquant un savoir. Nous nous sommes concentrés sur les manifestations (i) d'accord ou (ii) de désaccord qui suivent systématiquement les contributions définitoires :

- Les définitions formulées par des « experts » (extrait 1) auxquels les participants attribuent normativement un statut épistémique supérieur sont susceptibles de trouver l'accord des participants. De même, les définitions, qui contrairement à l'acception traditionnelle du concept - sont à la fois sémantiquement vagues (p. ex. "le sfc [...] il y a beaucoup de différentes pathologies » (extrait 2) ; «c'est un quelque chose d'individuel » (extrait 5)) et présentées 
comme étant ancrées dans l'expérience personnelle des personnes qui les formulent, donnent souvent lieu à des manifestations d'accord. Alors que ces définitions ne seraient d'aucune utilité dans les domaines « traditionnels » de la lexicographie et de la terminographie, elles révèlent leur utilité pratique dans les interactions soumises à examen : en effet, elles permettent aux interlocuteurs de se catégoriser mutuellement comme souffrant de la "même » maladie et donc comme étant des membres légitimes du groupe d'entraide.

- En revanche, sont fréquemment suivies d'un désaccord les pratiques définitoires qui non seulement instaurent une équivalence entre deux entités (p. ex. " le sfc c'est [...] tu as dépassé les limites et tu ne peux plus revenir en arrière » (extrait 3) ; le sfc «c'est lyme » (extrait 6)) mais qui sont présentées et/ou traitées comme étant valables pour tous les souffrants du SFC et, par conséquent, pour tous les co-participants. C'est surtout dans l'expression du désaccord que les participants rendent pertinentes des dissymétries épistémiques, allant jusqu'à la remise en cause de l'autorité épistémique d'un participant, et donc de son « droit à définir»".

Au vu de nos analyses, les participants s'engageant dans des pratiques définitoires ne se limitent pas à remplir de " sens » un nom tel que SFC : ils négocient aussi les droits et les obligations qui sont liés à de telles pratiques. Celle ou celui qui accomplit une pratique définitoire est tenu(e) responsable (accountable) pour celle-ci et doit par conséquent défendre son point de vue : l'exhibition d'un savoir est une ressource fondamentale à ce propos. Les pratiques définitoires n'entraînent pas uniquement une catégorisation de la maladie mais aussi de l'auteur de la définition.

D'un point de vue formel, nous avons observé de nombreuses définitions dichotomiques, qui réifient le principe classique de l'équivalence entre definiens et definiendum. Toutefois, et c'est la raison pour laquelle nous préférons parler de pratiques définitoires, les définitions ne sont pas toujours délivrées " en bloc ", en une seule fois : $l^{\prime}$ occurrence d'une définition donnant lieu à une exhibition de (dés)accord, l'absence de toute manifestation de (dés)accord, etc. peuvent engendrer une expansion, une réorientation d'une définition initiale (extrait 6) ; de même, une définition entraînant d'abord un désaccord peut être transformée au cours de l'interaction jusqu'à rendre finalement possible l'accord des interlocuteurs (extraits 3-5).

Ainsi, en nous focalisant sur les pratiques définitoires, nous avons contribué à la description d'un concept linguistique et philosophique qui avait été traité de façon désincarnée dans les précédentes recherches. D'un point de vue interactionnel, nous avons contribué à l'analyse d'environnements jusqu'ici peu étudiés en analyse conversationnelle, comme les groupes d'entraide pour maladies chroniques. Nous espérons enfin que cet article pourra donner lieu à une meilleure compréhension des problèmes auxquels les souffrants de SFC font face jour après jour. 


\section{CONVENTIONS DE TRANSCRIPTION}

\begin{tabular}{|c|c|}
\hline & intonation conclusive \\
\hline & intonation continuative \\
\hline ? & intonation interrogative \\
\hline (.) & micro-pause non mesurée \\
\hline$(0.3)$ & pause mesurée en secondes \\
\hline plutôt & emphase \\
\hline & allongement \\
\hline parl- & troncation \\
\hline UI & voix haute \\
\hline${ }^{\circ}$ oui $^{\circ}$ & voix basse \\
\hline${ }^{\circ}{ }^{\circ} \mathrm{Oui}^{\circ \circ}$ & voix très basse, chuchoté \\
\hline$<>$ & débit ralenti \\
\hline$<$ & débit accéléré \\
\hline. $\mathrm{h}$ & aspiration \\
\hline h & expiration \\
\hline [ ] & début et fin de chevauchement \\
\hline & enchaînement rapide \\
\hline (alors) & $\begin{array}{l}\text { continuation du tour de parole au delà de la fin de la ligne } \\
\text { transcription incertaine }\end{array}$ \\
\hline & segment incompréhensible pour le transcripteur \\
\hline
\end{tabular}

\section{Références}

ARISS S. M. (2009), “Asymmetrical knowledge claims in general practice consultations with frequently attending patients: Limitations and opportunities for patient participation", Social Science \& Medicine 69 (6), 908-919.

ARMInEN I. (1998), Therapeutic Interaction. A Study of Mutual Help in the Meetings of Alcoholic Anonymous, Helsinki: Finnish Foundation for Alcoholic Studies.

BaR-HILlel Y. (1954), “Indexical expressions”, Mind 63 (251), 359-379.

BEACH W. (ed.) (2001), “Introduction: Diagnosing 'lay diagnosis”', Text \& Talk 21 (1/2), 13-18.

BüLow P. H. (2004), “Sharing experiences of contested illness by storytelling”, Discourse \& Society 15 (1), 33-53.

CLEMENTS A. et al. (1997), "Chronic fatigue syndrome: a qualitative investigation of patients' beliefs about the illness”, Journal of Psychosomatic Research 42 (6), 615-624.

De Fornel M. \& Verdier M. (2014), Aux Prises avec la douleur. Analyse conversationnelle et consultations d'analgésie, Paris : Éditions de l'EHESS.

De Stefani E., Sambre P. \& Van De Mieroop D. (2016), “The interactional history of examples and parentheses: Note-taking practices in multiparty interaction among attendees of a mutual-help group for Chronic Fatigue Syndrome (CFS) sufferers", Language and Dialogue 6 (1), 110-139.

DepPermann A. (2011a), "The study of formulations as a key to an interactional semantics", Human Studies 34, 115-128. 
DePPERMANN A. (2011b), “Notionalization: The transformation of descriptions into categorizations", Human Studies 34, 155-181.

Deppermann A. (2015), "Wissen im Gespräch : Voraussetzung und Produkt, Gegenstand und Ressource ", InList 57. [http://www.inlist.uni-bayreuth.de/issues/57/inlist57.pdf]

DePPERMANN A. (2016), "La définition comme action multimodale pour des enjeux pratiques: définir pour instruire à l'auto-école ", Langages 204. (ce volume)

DREW P. \& HOLT E. (1998), "Figures of speech: Figurative expressions and the management of topic transition in conversation", Language in Society 27 (4), 495-522.

FREGE G. (1892), "Über Sinn und Bedeutung ", Zeitschrift für Philosophie und philosophische Kritik, Neue Folge 100, 25-50.

FUKUDA K. et al. (1994), “The chronic fatigue syndrome”, Annals of Internal Medicine 121, 953-959.

Garfinkel H. (1967), Studies in Ethnomethodology, Englewood Cliffs (NJ): Prentice-Hall.

GILL V. T. \& RoberTs F. (2013), “Conversation analysis in medicine”, in J. Sidnell \& T. Stivers (eds), The Handbook of Conversation Analysis, Malden (MA): Blackwell, 575-592.

GRECO L. (2016), " Définir le genre et la parenté en contexte LGBTQ : la définition comme laboratoire catégoriel et comme performance ", Langages 204. (ce volume)

GRUE J. (2014a), "A garden of forking paths: A discourse perspective on 'myalgic encephalomyelitis' and 'chronic fatigue syndrome"', Critical Discourse Studies 11 (1), 35-48.

GRUE J. (2014b), “Technically disabled, ill for all practical purposes? Myalgic encephalopathy/chronic fatigue syndrome discourse in Norway", Disability \& Society 29 (2), 213-223.

HALONEN M. (2008), "Person reference as a device for constructing experiences as typical in group therapy”, in A. Peräkylä et al. (eds), Conversation Analysis and Psychotherapy, Cambridge: Cambridge University Press, 139-151.

Heritage J. (2012), “Epistemics in action: Action formation and territories of knowledge", Research on Language and Social Interaction 45 (1), 1-29.

Heritage J. \& MAYNARD D. W. (2006), “Problems and prospects in the study of physician-patient interaction: 30 years of research", Annual Review of Sociology 32, 351-374.

HERITAGE J. \& RAYMOND G. (2005), “The terms of agreement: indexing epistemic authority and subordination in assessment sequences", Social Psychology Quarterly 68, 15-38.

HORTON-SALWAY M. (2001), "Narrative identities and the management of personal accountability in talk about ME: A discursive psychology approach to illness narrative", Journal of Health Psychology 6 (2), 247-259.

HORTON-SALWAY M. (2002), “Bio-psycho-social reasoning in GPs' case narratives: The discursive construction of ME patients' identities", Health: An Interdisciplinary Journal for the Social Study of Health, IIIness and Medicine 6 (4), 401-421.

IOM (INSTITUTE OF MEDICINE) (2015), Beyond Myalgic Encephalomyelitis/Chronic Fatigue Syndrome. Redefining an Illness, Washington: National Academies Press.

JEFFERSON G. (2004), “Glossary of transcript symbols with an introduction”, in G. H. Lerner (ed.), Conversation Analysis. Studies from the First Generation, Amsterdam/Philadelphia: John Benjamins, 13-31.

KLEIBER G. (1990), La Sémantique du prototype, Paris : Presses Universitaires de France.

LEHTINEN E. (2013), “Hedging, knowledge and interaction: Doctors' and clients' talk about medical information and client experiences in genetic counseling", Patient Education \& Counseling 92 (1), 31-37. 
Définir les mots dans l'interaction : un essai de sémantique interactionnelle

MACMARTIN C. \& LEBARON C. (2006), "Multiple involvements within group interaction: A videobased study of sex-offender therapy", Research on Language and Social Interaction 39 (1), 41-80.

MARTIN R. (1990), "La définition naturelle ", in J. Chaurand \& F. Mazière (éds), La Définition, Paris : Larousse, 86-95.

MILL J. S. (1843), A System of Logic, Ratiocinative and Inductive, London: John W. Parker.

PotTIER B. (1963), Recherches sur l'analyse sémantique en linguistique et en traduction mécanique, Nancy : Université de Nancy.

REY-DEBOVE J. (1971), Étude linguistique et sémiotique des dictionnaires français contemporains, Paris/La Haye : Mouton.

Robinson R. (1962), Definition, Oxford: Clarendon Press.

SACHS L. (2001), "From a lived body to a medicalized body: Diagnostic transformation and chronic fatigue syndrome", Medical Anthropology 19 (4), 299-317.

SACKS H. (1972), "On the analyzability of stories by children", in J. Gumperz \& D. Hymes (eds), Directions in Sociolinguistics. The Ethnography of Communication, New York: Rinehart \& Winston, 325-345.

SAMBRE P. (2005), Émergence et conceptualisation de la définition en langue naturelle. Une étude de cas sur "Internet " en néerlandais et en français, $\mathrm{PhD}, \mathrm{KU}$ Leuven.

SCHEGLOFF E. \& SACKS H. (1973), “Opening up closings”, Semiotica 8 (4), 289-327.

SHARPE M. C. et al. (1991), "A report. Chronic fatigue syndrome: Guidelines for research", Journal of the Royal Society of Medicine 84, 118-121.

Traverso V. \& Ravazzolo E. (2016), " Définitions ostensives co-construites. Le cas de la visite guidée ", Langages 204. (ce volume)

TUCKER I. (2004), “'Stories' of chronic fatigue syndrome: An exploratory discursive psychological analysis", Qualitative Research in Psychology 1, 153-167.

WATSON R. D. (1978), "Categorization, authorization and blame-negation in conversation", Sociology 12, 105-113.

Whitehead A. \& Russell B. (1910-1913), Principia mathematica, Cambridge: Cambridge University Press.

WittgensteIn L. (1953), Philosophische Untersuchungen, Oxford: Blackwell. 